\title{
Concomitant Use of Cyclobenzaprine and Psychotropic Medications
}

\author{
Nevio Cimolai
}

\begin{abstract}
Cyclobenzaprine has been cited as a co-factor or cause in serotonin syndrome following co-ingestion of other psychotropic medications. An observational study was conducted for a cumulative consumption of this agent among patients for 24 years, 8 months; no evidence of serotonin syndrome or malignant neuroleptic disorder was apparent despite a variety of concomitant psychotropic medications used.
\end{abstract}

Keywords: Cyclobenzaprine; Serotonin; Serotonin syndrome; Tricyclic; Toxicity

\section{Introduction}

Cyclobenzaprine is a commonly used tricyclic pharmacological agent which has reasonably good tolerance and safety profiles [1]. It is commonly used for muscle relaxation in the context of acute and/or chronic neck and back pain but has also been used as treatment for fibromyalgia. It frequently causes drowsiness and may facilitate sleep.

Keegan et al. reported two patient case studies in which it was postulated that cyclobenzaprine use in the midst of other serotoninergic drug ingestion led to a serotonin syndrome [2]. Day and Jeanmonod subsequently published another similar case report [3]. Despite the widespread use of

Manuscript accepted for publication June 23, 2011

The University of British Columbia, Vancouver, British Columbia, Canada; Children's and Women's Health Centre of British Columbia Vancouver, British Columbia, Canada V6H 3V4. Email: ncimolai@ interchange.ubc.ca

doi:10.4021/jmc241w either cyclobenzaprine or serotoninergic agents, there is a paucity of data on the potential toxicity of concomitant use including serotonin syndrome. This review sought to prospectively determine the risk of such concomitant use in an outpatient setting.

\section{Case Series}

A prospective follow-up in a community general practice setting was conducted for 23 patients (12 male, 11 female) who had consumed cyclobenzaprine in tandem with other psychotropic medications over the years 2005-2011. Ages ranged from 21-55 years (mean 44.3 years). The total time period of patient-year observation amounted to approximately 24 years, 8 months. The duration of simultaneous drug use for any one patient ranged from 5 days to 5 years, 2 months (mean 12.9 month). Eleven of 23 patients were to be continuously prescribed cyclobenzaprine due to its beneficial effect.

All patients received cyclobenzaprine as part of a pain management strategy: 12 with back pain, 6 with neck and shoulder pain, and 5 various other (generalized chronic pain, fibromyalgia, hip pain, and muscle spasm after other drug withdrawal). Eleven patients received $10 \mathrm{mg}$ nightly doses only, whereas the others consumed multiple doses per day ranging from $10-80 \mathrm{mg}$ per day (mean $20.9 \mathrm{mg} /$ day). These individuals were receiving multiple other medications (range 2-7 per patient) which included one or more psychotropic drugs (range 1-4 per patient). Twenty-two of 23 patients reported a clinically beneficial effect of cyclobenzaprine use including four who reported a positive effect on sleep. The psychotropic medications used for these patients included amitriptyline, benzodiazepines, bupropion, citalopram, escitalopram, gabapentin, lithium, methotrimeprazine, mirtazapine, olanzapine, quetiapine, risperidone, trazodone, venlafaxine, and zopiclone. There was no evidence of any serotoninergic side effect despite the lengthy experience. Seven of 23 patients reported sedation as a side effect, and one patient purposely reduced the dose of cyclobenzaprine as a result. There were no side effects that prompted cessation of either cyclobenzaprine or any other medication. In addition to the aforementioned psychotropic agents, some 
patients were also ingesting codeine, tramadol, methadone, hydromorphone, carbamazepine, and benztropine.

\section{Discussion}

Serotonin syndrome can constitute a severe medical emergency. Symptoms may include agitation, tachycardia, acute hypertension, hyperthermia, diaphoresis, diarrhea, tremor, hyperreflexia, clonus, muscle rigidity, and mydriasis. The observed severity, however, can range from mild to severe including, but rarely, death. The diagnosis can be confusing to the uninitiated. This adverse reaction usually occurs abruptly. Although reported to occur with proserotoninergic drugs (conventionally thought of as monoamine oxidase inhibitors and selective serotonin reuptake inhibitors but including other antidepressants), there have been citations associated with drugs such as opiates and opiate analogues, lithium, triptans, amphetamines, among many others [3-5]. Although the pathology is reversible after drug discontinuation, it might be anticipated that the experience with serotonin syndrome might increase in this era of polypharmacy.

There is a dearth of publications regarding co-ingestion of cyclobenzaprine and other psychotropic medications. Cantini et al. describe a small group of fibromyalgic patients who were randomized to fluoxetine and cyclobenzaprine; no episodes of toxicity were indicated [6]. The case series detailed herein demonstrates the lack of adverse cyclobenzaprine interactions with a wide array of psychotropic pharmacological agents despite observation over a lengthy span of patient-year consumption.

The number of previous citations of cyclobenzaprine causing serotonin syndrome are very few [2, 3]. Gillman rightfully cautions against overinterpretation of this association [7]. For example, with variable manifestations, one may find it difficult to differentiate the cited case reports from the single case study of malignant neuroleptic syndrome with cyclobenzaprine use [8]. Boyer and Shannon comment on the basis for differentiation of similarly appearing clinical entities [4]. Given the frequency of cyclobenzaprine use and as well the widespread use of serotoninergic medications of several classes, one would have anticipated more frequent reports of serotonin syndrome. A review of several databases did not yield any further such documentations.

Citations of cyclobenzaprine-associated serotoninergic syndrome are extremely rare. Our case series of cyclobenzaprine use in the midst of co-administration with other psychotropic agents over an accumulation of some 24-25 patient years did not provide evidence of such a reaction. The cumulative experience therefore suggests cautious optimism in the co-administration of cyclobenzaprine with serotoninergic medications. Our series did not include patients who co-consumed monoamine oxidase inhibitors, and therefore care should continue to be exercised when the latter use preexists.

\section{References}

1. Cimolai N. Cyclobenzaprine: a new look at an old pharmacological agent. Expert Rev Clin Pharmacol. 2009;2:255-263.

2. Keegan MT, Brown DR, Rabinstein AA. Serotonin syndrome from the interaction of cyclobenzaprine with other serotoninergic drugs. Anesth Analg. 2006;103(6):1466-1468.

3. Day LT, Jeanmonod RK. Serotonin syndrome in a patient taking Lexapro and Flexeril: a case report. Am J Emerg Med. 2008;26(9):1069 e1061-1063.

4. Boyer EW, Shannon M. The serotonin syndrome. N Engl J Med. 2005;352(11):1112-1120.

5. Gillman PK. A review of serotonin toxicity data: implications for the mechanisms of antidepressant drug action. Biol Psychiatry. 2006;59(11):1046-1051.

6. Cantini F, Bellandi F, Niccoli L, Di Munno O. Fluoxetin combined with cyclobenzaprine in the treatment of fibromyalgia. Minerva Med. 1994;85(3):97-100.

7. Gillman PK. Is there sufficient evidence to suggest cyclobenzaprine might be implicated in causing serotonin toxicity? Am J Emerg Med. 2009;27(4):509510; author reply 510.

8. Theoharides TC, Harris RS, Weckstein D. Neuroleptic malignant-like syndrome due to cyclobenzaprine? J Clin Psychopharmacol. 1995;15(1):79-81. 